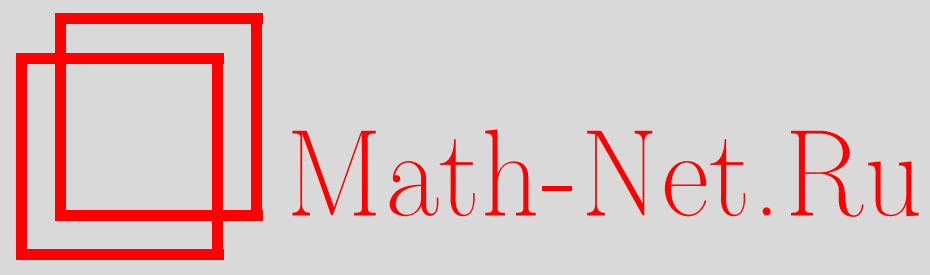

В. А. Клепцын, Б. А. Рабинович, Аналитическая классификация фуксовых особых точек, Матем. заметки, 2004, том 76 , выпуск 3, 372-383

DOI: https://doi.org/10.4213/mzm114

Использование Общероссийского математического портала Math-Net.Ru подразумевает, что вы прочитали и согласны с пользовательским соглашением http://www . mathnet.ru/rus/agreement

Параметры загрузки:

IP: 54.166 .219 .16

26 апреля 2023 г., 14:39:35

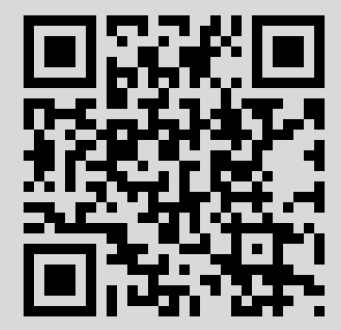




\title{
АНАЛИТИЧЕСКАЯ КЛАССИФИКАЦИЯ ФУКСОВЫХ ОСОБЫХ ТОЧЕК
}

\author{
В. А. Клепцын, Б. А. Рабинович
}

В работе исследуется задача локальной аналитической классификации фуксовых особых точек. Хорошо известны вид резонансной формальной нормальной формы $(\Phi H \Phi)$ системы с фуксовой особой точкой и утверждение о локальной аналитической эквивалентности системы своей резонансной $\Phi H \Phi$. Однако некоторые различные резонансные $\Phi H \Phi$ могут быть локально аналитически эквивалентны друг другу. Основной результат работы - теорема, сводящая вопрос о локальной аналитической эквивалентности резонансных $\Phi H \Phi$ к вопросу о сопряженности некоторых построенных по этим $\Phi H \Phi$ матриц (оказывающихся нильтреугольными) блочноверхнетреугольной матрицей. Как следствие, проблема локальной аналитической классификации фуксовых особых точек сводится к исследованию орбит действия группы блочноверхнетреугольных матриц на нильтреугольных сопряжениями.

Библиография: 13 названий.

\section{1. Введение}

1.1. Постановка задачи. Рассмотрим систему с фуксовой особой точкой (здесь и далее мы считаем особой точкой точку $t=0$ )

$$
\dot{z}=\frac{A(t)}{t} z, \quad z \in \mathbb{C}^{N}, \quad t \in \mathbb{C}
$$

где матрица $A(t)$ голоморфна в некоторой окрестности нуля.

По определению (см. работу В.И. Арнольда и Ю.С. Ильяшенко [1]) две линейных системы локально аналитически (или голоморфно) эквивалентны, если одна из них переводится в другую линейной по неизвестному заменой $z=H(t) w$, где матрища $H(t)$ голоморфна вместе со своей обратной в некоторой окрестности особой точки.

Задача, исследованию которой посвящена статья - получение аналитической классификации фуксовых особых точек и исследование связанного с этим вопроса: проблемы локальной аналитической эквивалентности фуксовых систем.

В работах А. Х. М. Левеля [2], Ф.Р. Гантмахера [3], Ю. С. Ильяшенко [4] описана резонансная формальная нормальная форма фуксовой системы и доказана аналитическая

Работа выполнена при частичной поддержке Российского фонда фундаментальных исследований, гранты № 02-01-00482, № 02-01-22002, фонда CRDF, грант RM1-2358-MO-02, и программы PICS "Singularities of differential equations, integrable systems and quantum groups" (CNRS). 
эквивалентность системы ее резонансной формальной нормальной форме $(\Phi H \Phi)$. Тем самым достаточно исследовать вопрос о сопряженности и задачу классификации для систем, приведенных к резонансным $\Phi$ $\Phi$.

Основной результат статьи, сформулированньй ниже (теорема 1), сводит рассматриваемые задачи к чисто алгебраическим, а именно, к вопросу о сопряженности некоторых матриц, построенных по системе, матрицей специального блочного вида, и к классификации орбит этого действия - точную формулировку см. в п. 1.5.

Идеи, ведущие к этому результату, присутствуют в работах А.А. Болибруха [5], Ф.P. Гантмахера [3], Д. В. Аносова и А. А. Болибруха [6]. Однако нам не удалось найти источника, где вопрос ставился бы именно в таком контексте (эквивалентности двух нормальных (форм); в то же время, получающийся ответ оказьвается простьгм и, как нам кажется, довольно красивым. Поэтому, понимая, что как технически, так и идейно теорема 1 не представляет сложности, мы, тем не менее, считаем ее одним из двух основных результатов настоящей работы.

Вторьм основным результатом работы является пример, показьвающий, что начиная с размерности 6 в задаче классификации фуксовых особых точек возникают модули. Именно, появляются семейства классов эквивалентности, зависящие от вещественного параметра. Точную формулировку см. в п. 1.5 (следствие 2).

Существование примера такого рода в алгебраической версии задачи следует из работы В. В. Кашина [7]. В разделе 5 такой пример строится явно.

1.2. Структура статьи. Статья устроена следующим образом: в п. 1.3 описан вид резонансной $\Phi Н \Phi$ системы с фуксовой особой точкой. В п. 1.4 вводятся обозначения, определяются использующиеся далее термины и делаются простейшие замечания о рассматриваемой задаче. В п. 1.5 формулируются основные результаты работы. В п. 1.6 суммируются два подхода, ведущие к получению резонансной $\Phi H \Phi$ : с точки зрения теоремы Пуанкаре-Дюлака и с точки зрения фундаментальной матрищы решений.

В разделе 2 исследуется вид матрицы, сопрягающей две системы, приведенные к резонансной $\Phi$ $Ф$. В разделе 3 доказаны сформулированные во введении утверждения основной результат (теорема 1) и следствие 1 . В разделе 4 мы приводим уже известные результаты, касающиеся классификации орбит действия групш верхнетреугольных и унитреугольных матриц на нильтреугольных сопряжениями - в наших терминах, случай вполне резонансной системы без кратных собственных значений. В разделе 5 приведены примеры, в которых естественные инварианты - набор собственных значений и жорданова нормальная форма оператора монодромии - совпадают, а сами системы, тем не менее, не сопряжены; более того, оказывается, что дополнительньй инвариант в одном из примеров может принимать континуум различных значений.

1.3. Вид резонансной формальной нормальной формы. Для описания резонансной нормальной формы системы (1) разложим фазовое пространство $\mathbb{C}^{n}$ в прямую сумму корневых для $A(0)$ подпространств. При блочном разбиении, соответствующем этому разложению в прямую сумму (с некоторым специальньм порядком блоков - см. 
ниже), матрища резонансной нормальной формы принимает вид

$$
\begin{gathered}
\dot{z}=\frac{B(t)}{t} z, \quad B(t)=\left(\begin{array}{cccc}
B_{11} & B_{12}(t) & \ldots & B_{1 s}(t) \\
0 & B_{22} & \ldots & B_{2 s}(t) \\
\vdots & \ddots & \ddots & \vdots \\
0 & \ldots & 0 & B_{s s}
\end{array}\right), \\
B_{i j}(t)=B_{i j}^{0} t^{\lambda_{i}-\lambda_{j}}, \\
B_{i j}^{0} \equiv \text { const . }
\end{gathered}
$$

У матрицы $B_{i i}$ есть единственное собственное значение $\lambda_{i}$, все $\lambda_{i}$ различны. Кроме того, $B_{i j}^{0} \neq 0$, только если $\lambda_{i}-\lambda_{j}$ - натуральное число или $i=j$. При этом блоки упорядочены так, что если $\lambda_{i}-\lambda_{j}$ - натуральное число, то $i<j$. Наконец, все матрищы $B_{i i}$ приведены к жордановой нормальной форме.

Назовем блочное разбиение, возникающее из разбиения $\mathbb{C}^{n}$ в прямую сумму корневых для $A(0)$ подпространств, корневым разбиением. Объединим в нем блоки, для которых $\lambda_{i}$ отличаются на целые числа. Мы получили более крупное блочное разбиение, которое будем назьвать резонансным. Далее, говоря о корневом разбиении, мы будем считать порядок блоков следуюшим: резонансные блоки упорядочены произвольным, но одним и тем же для всех систем с данным набором собственных значений способом. Внутри каждого резонансного блока корневые блоки упорядочены по убыванию вещественной части собственного значения.

Из предыдущего описания следует, что матрица резонансной $\Phi$ $\Phi$ блочнодиагональна в смысле резонансного и блочноверхнетреугольна в смысле корневого разбиений, причем каждьй ее резонансньй блок имеет вид (2). Это и есть окончательное описание резонансной $\Phi Н \Phi$.

В книге Гантмахера [3] приведена $\Phi H \Phi$, отличающаяся от описанной дополнительными упрощениями. Впрочем, поскольку для наших целей достаточно вида (2), мы эти упрощения выполнять не будем.

1.4. Простейшие замечания. Обозначения. Будем назьвать форму, описанную в предыдушем пункте, предварительной нормальной формой. Несложно видеть, что при голоморфной замене матрища $A(0)$ просто сопрягается матрищей $H(0)$. Собственные значения $\lambda_{i}$ матрицы-вычета $A(0)$, являющиеся инвариантами аналитического сопряжения, называются собственными значениями системы.

По определению система называется вполне резонансной, если все ее собственные значения различаются на целые числа. Из вида предварительной нормальной формы (2) следует, что каждая система с фуксовой особой точкой локально аналитически эквивалентна прямой сумме вполне резонансных. Несмотря на это, пока что мы не можем ограничиться рассмотрением только их: ниоткуда не следует, что сопряжение двух прямых сумм тоже есть прямая сумма сопряжений, а, значит, прямые суммы неэквивалентных вполне резонансных подсистем могут оказаться эквивалентны. Впрочем, для случая, когда резонансов нет, т.е. когда любые два собственных значения не отличаются на целое число, отсюда следует известное (см. работу Э.А. Коддингтона и Н. Левинсона [8]) утверждение: нерезонансная система локально аналитически эквивалентна системе

$$
\dot{z}=\frac{A(0)}{t} z
$$


и, следовательно, сопряженность матриц $A(0)$ является критерием для сопряженности систем.

Кроме набора собственных значений, у системы есть еще один естественньй инвариант - оператор монодромии, рассматриваемьй с точностью до сопряжения. Однако, как будет показано ниже, эта система инвариантов далеко не полна.

Будем говорить, что матрища $M$ блочноверхнетреугольная правильной структу$p b l$, если она блочнодиагональна в смысле резонансного и блочноверхнетреугольна в смысле корневого разбиений. Поскольку далее эти разбиения будут совпадать для всех рассматриваемых систем (в силу совпадения наборов собственных значений), мы будем говорить о правильной структуре матрицы, не уточняя, относительно какой именно из матриц-вычетов эта структура рассматривается.

Обозначим $\widehat{B}(t)=B(t)-\operatorname{diag}(B)$ для матрицы $B(t)$ системы в предварительной нормальной форме (здесь $\operatorname{diag}(B)$ - диагональная матрица, на диагонали совпадающая с $B$ ), и назовем $\widehat{B}(1)$ характеристической матрицей системы (2). Кроме того, обозначим через $m_{j}$ кратность собственного значения $\lambda_{j}$, и пусть $k_{i j}=\lambda_{i}-\lambda_{j}$. При этом выполнено $k_{i j}+k_{j l}=k_{i l}$ при всех $i, j, l$.

\section{5. Формулировка основных результатов.}

ТЕОРема 1 (основной результат). Две системы, приведенные к предварительной нормальной форме (2), голоморфно әквивалентны тогда и только тогда, когда у них одинаковые наборы собственных значений и их характеристические матрицы сопряжены некоторой блочноверхнетреугольной матричей правильной структу$p b l$.

В частности, эта теорема утверждает, что после приведения систем к предварительной нормальной форме вопрос об их эквивалентности можно заменить вопросом о поблочной эквивалентности - об эквивалентности вполне резонансных подсистем. Тем самым, задача о классификации предварительных нормальных форм фуксовых систем сводится к задаче о классификации блочноверхнетреугольных матриц с нильтреугольными блоками-матрицами специального вида (нильпотентная жорданова нормальная форма) по диагонали под действием сопряжения блочноверхнетреугольньми матрицами.

Для случая, когда среди собственных значений матрищы $B(0)$ нет кратных (все блоки размера 1), эта теорема была доказана в работе Б. А. Рабиновича [9].

СлЕДСТВИЕ 1. Проблема локальной аналитической әквивалентности двух систем с фуксовыми особыми точками алгебраически разрешима.

Алгебраическая версия нашей задачи - классификация орбит действия блочноверхнетреугольных матриц на блочнонильтреугольных сопряжениями - давно известна и хорошо исследована. Далее мы приведем некоторые известные результаты для наиболее простого нетривиального случая - когда среди собственных значений систем нет кратных (при этом собственные значения системы могут отличаться на целое число, но не совпадать). Используя эти результаты, мы покажем, что число классов сопряженности систем достаточно большой размерности с даннњм набором собственных значений для некоторых наборов оказывается бесконечньм. 
СлЕДСТВИЕ 2 (основной результат № 2). Начиная с размерности 6, для некоторых наборов собственных значений ( а именно, для тех, среди которых по меньшей мере 6 отличающихся на иелье числа, но при этом попарно различных) число классов голоморфной сопрязсенности систем с фуксовой особой точкой бесконечHO.

1.6. Другое описание предварительной нормальной формы. Эквивалентность всякой системы (1) некоторой системе вида (2) может быть получена двумя способами. Первьй из них (см. работы [3], [4]) - линейная версия теоремы Пуанкаре-Дюлака, применяемая к системе

$$
\left\{\begin{array}{l}
\dot{z}=B(t) z \\
\dot{t}=t
\end{array}\right.
$$

При этом резонансами оказываются соотношения $\lambda_{i}=\lambda_{j}+k \cdot 1$; здесь 1 - собственное значение, соответствующее оси $t$. В результате получается формальная эквивалентность двух систем, после чего доказьвается сходимость ряда для сопрягающего отображения. Этот способ прямо приводит к матрице системы вида (2).

Предварительная нормальная форма может быть также получена (см. работу Левеля [2]), исходя из фундаментальной матрицы решений. Приведем здесь (слегка видоизмененное) рассуждение из [2], доказывающее это. Согласно теореме Левеля (см. [2], а также изложение в книге Болибруха [5, теорема 1, с. 47]), в окрестности фуксовой особой точки 0 найдется $\Phi \mathrm{MP}$, соответствующая некоторому базису в пространстве решений, имеющая вид

$$
Z(t)=U(t) t^{N} t^{E}
$$

Здесь матрица $U(t)$ голоморфно обратима в окрестности особой точки. Матрица $E$ верхнетреугольная, имеющая блочнодиагональньй (в смысле некоторого разбиения; см. ниже) вид, причем в каждом блоке у $E$ есть только одно собственное значение $\rho_{k}$ и выполнено $\operatorname{Re} \rho_{k} \in[0,1)$. Наконец, $N$ - диагональная матрища, по диагонали которой стоят целые числа (нормирования), невозрастающие в каждом блоке матрицы $E$.

Сделаем замену, выбрав в качестве матрицы замены $H(t)$ как раз матрицу $U(t)$. После замены получаем $\Phi$ МР в виде

$$
W(t)=t^{N} t^{E}
$$

Тогда матрица системы равна

$$
\frac{B(t)}{t}=\dot{W} W^{-1}=\frac{N+t^{N} E t^{-N}}{t} .
$$

Как легко видеть, эта матрища имеет вид (2), где корневое блочное разбиение получено объединением блочного разбиения матрицы $E$ с блочньм разбиением матрицы $N$ на скалярные блоки. Собственные значения $B(0)$ - суммы нормирований и соответствующих им собственных значений матрицы $E$. При этом получаем, что вьшеупомянутое блочное разбиение матрищы $E$ (в котором она блочнодиагональна) - это и есть резонансное разбиение.

Обратно, как показано в работе Ильяшенко [4], для системы вида (2) ФМР имеет вид (3). 
В дальнейшем для доказательства всех утверждений работы мы будем использовать именно подход с точки зрения $Ф \mathrm{MP}$, хотя стоит отметить, что именно подход с точки зрения теоремы Пуанкаре-Дюлака позволяет алгоритмизовать процедуру приведения к предварительной нормальной форме.

\section{2. Вид матрицы сопрягающей замены}

Вопросы, связанные с утверждениями этого раздела, обсуждались в работах Аносова и Болибруха [6], Болибруха [5], [10], Гантмахера [3]. Однако, поскольку нам не удалось найти ссылку, где рассматривается вопрос именно в этой его постановке, для полноты изложения мы приводим следуюшие утверждения вместе с их доказательствами.

ЛЕмма 1. Пусть голоморфно обратимая в проколотой окрестности 0 и мероморфная в 0 матрииа $H(t)$ сопрягает две системы вида (2) с одинаковыми наборами собственных значений (с учетом кратности). Тогда при корневом разбиении $H(t)=\left(H_{i j}(t)\right)$ получаем

$$
H_{i j}(t)=H_{i j}^{0} t^{k_{i j}}, \quad H_{i j}^{0} \equiv \text { const }
$$

причем $H_{i j}^{0} \neq 0$, только если $k_{i j}=\lambda_{i}-\lambda_{j} \in \mathbb{Z}$.

ДокАЗАТЕльСтво. Матрица $H(t)$ сопрягает две системы тогда и только тогда, когда она переводит $\Phi \mathrm{MP} \mathrm{одной} \mathrm{системы} \mathrm{в} \mathrm{(некоторую)} \Phi \mathrm{MP} \mathrm{другой.} \mathrm{Две} \Phi \mathrm{MP} \mathrm{одной} \mathrm{и} \mathrm{той}$ же системы отличаются умножением на постоянную матрицу, поэтому $H(t)$ сопрягает две системы в нормальной форме Левеля с $\Phi \mathrm{MP} t^{N} t^{E}$ и $t^{N^{\prime}} t^{E^{\prime}}$ тогда и только тогда, когда

$$
\exists V: \quad H(t) t^{N} t^{E}=t^{N^{\prime}} t^{E^{\prime}} V .
$$

Тогда

$$
t^{-N^{\prime}} H(t) t^{N}=t^{E^{\prime}} V t^{-E}
$$

Заметим, что элементы $t^{E^{\prime}}$ - линейные комбинации $t^{\rho_{\alpha}^{\prime}} \ln ^{k} t$, где $\rho_{\alpha}^{\prime}-$ собственные значения $E^{\prime}$, a $k \in \mathbb{N} \cup\{0\} ;$ элементы $t^{-E}$ - линейные комбинации $t^{-\rho_{\alpha}} \ln ^{k} t$, где $\rho_{\alpha}-$ собственные значения $E$. Отсюда элементы матрицы, стоящей в правой части (5), есть линейные комбинации $t^{\rho} \ln ^{k} t$, где $\rho$ представляется в виде разности собственных значений $E^{\prime}$ и $E$. Поскольку вещественные части собственньх значений $E$ и $E^{\prime}$ лежат на $[0,1)$, имеем $\operatorname{Re} \rho \in(-1,1)$.

Матрица же в левой части (5) однозначна, поэтому в правой части допустимы лишш $t^{\rho} \ln ^{k} t$ c $\rho \in \mathbb{Z}, k=0$. Отсюда $(\operatorname{Re} \rho \in(-1,1) \Rightarrow \rho=0)$ матрица в правой части должна быть постоянной. Обозначим ее через $\widetilde{V}$.

Поскольку наборы собственных значений одинаковы, имеем $N=N^{\prime}, \operatorname{diag}(E)=$ $\operatorname{diag}\left(E^{\prime}\right)$. Кроме того, в резонансном разбиении блок $(\alpha, \beta)$ правой части $(5)$ имеет вид $t^{\rho_{\alpha}-\rho_{\beta}} P(\ln t)$, где $P$ - матричнозначный полином. Из-за однозначности $P \equiv 0$ при $\alpha \neq \beta$ (поскольку в этом случае $\rho_{\alpha}-\rho_{\beta} \notin \mathbb{Z}$ ). Отсюда матрица $\widetilde{V}$ блочнодиагональна в смысле резонансного разбиения.

Из формулы (5) имеем

$$
H(t)=t^{N} \widetilde{V} t^{-N}
$$


что означает для блоков (в смысле корневого разбиения) $H_{i j}$

$$
H_{i j}(t)=t^{n_{i}} \widetilde{V}_{i j} t^{-n_{j}}=t^{n_{i}-n_{j}} \widetilde{V}_{i j}=t^{\lambda_{i}-\lambda_{j}} \widetilde{V}_{i j}
$$

поскольку внутри одного вполне резонансного блока разность нормирований равна разности соответствующих собственных значений $B(0)$, а матрица $\widetilde{V}$ блочно-диагональна в смысле разбиения на вполне резонансные подсистемы.

ЗАмЕчАниЕ 1 . Подставив в формулу для $\widetilde{V}$ (правую часть уравнения (5)) значение $t=1$, получаем $\widetilde{V}=V$, так как $1^{M}=$ I для любой матрицы $M$. (Эта подстановка законна, так как равенство (5) есть равенство матриц с элементами - рациональными функциями, и потому из его выполнения в малой окрестности 0 следует его вьполнение при всех $t$ ).

СЛЕДСТВИЕ 3. Пусть голоморфно обратимая в окрестности 0 матрииа $H(t)$ сопрягает две системы вида (2). Тогда при блочном разбиении $H(t)=\left(H_{i j}(t)\right)$ получаем

$$
H_{i j}(t)= \begin{cases}H_{i j}^{0} t^{k_{i j}}, & \lambda_{i}-\lambda_{j} \in \mathbb{N} \cup\{0\}, \\ 0, & \lambda_{i}-\lambda_{j} \notin \mathbb{N} \cup\{0\} ;\end{cases}
$$

в частности, при всех $t$ матрица $H(t)$ блочноверхнетреугольная правильной структуры.

ДоКАЗАТЕЛЬСТВо. Если голоморфно обратимая матрица сопрягает две системы вида (2), то их матришы-вычеты в 0 сопряжены. Поэтому они обладают одинаковыми наборами собственных значений (с учетом кратности) - применима предыдущая лемма. Осталось показать, что при $\lambda_{i}-\lambda_{j}=k_{i j} \in(-\mathbb{N})$ имеем $H_{i j}^{0}=0$ : если это не так, то в силу формулы (4) матрица $H(t)$ не будет голоморфна в окрестности 0. Отсюда $H(t)-$ блочноверхнетреугольная матрища требуемого вида.

Следующее утверждение позволяет свести вопрос о сопряженности произвольных систем к сопряженности вполне резонансных.

СЛЕДСТВИЕ 4. При наличии собственных значений, отличающихся на нецелье числа, сопряжение происходит поблочно межсду вполне резонансными блоками-системами.

\section{3. Доказательство основного результата и следствия 1}

ДоКАЗАТЕЛЬСТВО ТЕОРЕМЫ 1 . Из формулы (6) и замечания 1 матрица замены имеет вид $H(t)=t^{N} V t^{-N}$. Подставив это в (5), получаем, что две системы в предварительной нормальной форме сопряжены такой заменой тогда и только тогда, когда

$$
\forall t \quad V=t^{E^{\prime}} V t^{-E}
$$

Это равносильно тому, что

$$
\forall t \quad V t^{E} V^{-1}=t^{E^{\prime}} \Longleftrightarrow \forall t \quad t^{V E V^{-1}}=t^{E^{\prime}} \Longleftrightarrow \forall t \quad V E V^{-1}=E^{\prime}
$$


Отсюда

$$
V E=E^{\prime} V
$$

В резонансном разбиении матрицы $V$ и $\operatorname{diag}(E)=\operatorname{diag}\left(E^{\prime}\right)$ блочнодиагональны, причем блоки $\operatorname{diag}(E)$ - скалярные матрицы. Значит, $V \operatorname{diag}(E)=\operatorname{diag}\left(E^{\prime}\right) V$. Следовательно, эквивалентным условием сопряженности является

$$
V(E-\operatorname{diag}(E))=\left(E^{\prime}-\operatorname{diag}\left(E^{\prime}\right)\right) V .
$$

Ho

$$
E-\operatorname{diag}(E)=E+N-\operatorname{diag}(E+N)=B(1)-\operatorname{diag}(B(1))=\widehat{B}(1)
$$

и, аналогично,

$$
E^{\prime}-\operatorname{diag}\left(E^{\prime}\right)=\widehat{C}(1)
$$

Кроме того, $V=H(1)$, так как $H(t)=t^{N} V t^{-N}$. Тем самым мы получили эквивалентное условие сопряженности в виде

$$
\widehat{B}(1) H(1)=H(1) \widehat{C}(1) .
$$

ДокАЗАТЕЛЬСТво СЛЕДСТвия 1. Поскольку предварительная нормальная формаэто формальная нормальная форма Пуанкаре-Дюлака с конечным числом резонансов (вида $\lambda_{i}=\lambda_{j}+k, k \in \mathbb{N}$ ), ее нахождение - алгебраически разрешимая задача (см. [3], где соответствующая процедура проведена явно).

В силу теоремы 1 задача сведена к вопросу о сопряженности двух матриц - $\widehat{B}(1)$ и $\widehat{C}(1)$ - матрицей некоторой блочной структуры.

Уравнение сопряженности $(\widehat{B}(1) M=M \widehat{C}(1))$ - линейное уравнение на $M$, поэтому, решив его в линейном пространстве матриц требуемой блочной структуры (не обязательно невырожденных), мы получаем некоторое его подпространство $L$, после чего исходный вопрос эквивалентен вопросу о наличии в $L$ невырожденной матрицы. Но $\operatorname{det} M-$ многочлен, поэтому вопрос о его обращении в тождественньй 0 на подпространстве $L$ алгебраически разрешим, что и доказывает утверждение следствия.

\section{4. Описание орбит действия группы верхнетреугольных матриц на нильтреугольных сопряжениями}

Приведенные в разделе сведения взяты из работ И. Б. Бродского [11], [12], В.В. Кашина [7].

Ограничимся случаем вполне резонансного простого спектра матрицы-вычета, когда алгебраическая версия вопроса звучит так: исследовать орбиты действия групшы вехрнетреугольных матриц на нильтреугольных сопряжениями.

Заметим, что сопряжение верхнетреугольной матрищей разбивается на два этапа: сопряжение унитреугольной и диагональной матрицами. При этом сопряжение диагональной матрицей не может изменить мест, на которых стоят ненулевые элементы - оно может только поменять сами эти элементы, оставляя их ненулевыми.

Упорядочим элементы нильтреугольной матрицы так, чтобы элементы с более "низких" диагоналей (с меньшей разностью $j-i$ ) были бы “старше" - имели бы большие 
номера, чем элементы с более “высоких" диагоналей; порядок элементов на одной диагонали неважен. Тогда действие унитреугольной матрищы сопряжением можно интерпретировать как действие некоторой унитреугольной матрицы порядка $N=n(n-1) / 2$ в линейном пространстве нильтреугольных матриц. Тем самым, задача сводится к описанию орбит действия некоторой подгрупшы Ли $G$ групшы унитреугольных матриц порядка $N$. В отношении таких действий в работах Бродского [11], [12] доказана следующая теорема.

ТЕОремА. Пусть есть подгруппа Ли $G$ группы унитреугольных матрии порядка $N$, естественным образом действующая на $\mathbb{R}^{N}$. Тогда каждая орбита $G$ содержит ровно один вектор $\left(\mu_{1}, \ldots, \mu_{N}\right)$ (нормальную форму) следующего вида: для любого $i, N \geqslant i \geqslant 1$, либо $\mu_{i}=0$, либо, действуя на $\mu$ теми әлементами $G$, которые не изменят $\mu_{N}, \ldots, \mu_{i+1}$, нельзя добиться обращения $\mu_{i}$ в 0. В последнем случае $\mu_{i}$ вообще нельзя изменить, сохранив $\mu_{N}, \ldots, \mu_{i+1}$.

Назовем носителем нильтреугольной матрицы множество номеров ненулевых элементов и будем говорить, что один носитель лексикографически старше другого, если для некоторого $1 \leqslant i \leqslant N$ все $j>i$ одновременно принадлежат или не принадлежат обоим носителям, а $i$ не принадлежит первому носителю и принадлежит второму (старшинство по отсутствию элементов).

В этих терминах эквивалентная формулировка теоремы звучит так.

ТеоремА. В каждой орбите действия $G$ есть единственный әлемент (нормальная форма) с лексикографически старшим носителем.

Легким следствием этой теоремы является следующее утверждение.

ЛЕмма 2. Носители нормальных форм двух матрии, сопряэсенных верхнетреугольной матрицей, совпадают, а сами эти нормальные формы сопряжены диагональной матричей.

ДоКАЗАТЕЛЬСТВо. Из сопряженности двух матриц верхнетреугольной матрицей следует, что их нормальные формы также сопряжены некоторой верхнетреугольной матрицей $T$. Пусть $F_{1}=T F_{2} T^{-1}$, где $F_{1}$ и $F_{2}$ - нормальные формы. Представим $T$ в виде $T=D U$, где $D$ - диагональная, $U$ - унитреугольная матрищы. Тогда $D^{-1} F_{1} D=$ $U F_{2} U^{-1}$. Носитель левой части совпадает с носителем $F_{1}$, а правая часть принадлежит орбите $F_{2}$ под действием $G$; следовательно, носитель $F_{1}$ лексикографически не старше носителя $F_{2}$. Аналогично, носитель $F_{2}$ лексикографически не старше носителя $F_{1}$. Тем самым эти носители совпадают. Но в силу единственности в орбите элемента с лексикографически максимальным носителем $U F_{2} U^{-1}=F_{2}$ (так как это элемент орбиты $F_{2}$ с тем же носителем), откуда $D^{-1} F_{1} D=F_{2}$.

Рассмотрим следуюший вопрос: сколько существует классов голоморфной эквивалентности фуксовых систем с данным набором собственньх значений? Для случая вполне резонансного набора различных собственных значений этот вопрос эквивалентен вопросу о числе классов эквивалентности нильтреугольных матриц под действием сопряжения блочноверхнетреугольными. В работе Кашина [7] показано, что в размерности $\leqslant 5$ число классов сопряженности конечно, и равно $1,2,5,16,61$ в размерностях $1,2, \ldots$, 5 соответственно, а при размерности $\geqslant 6$ оно бесконечно. Далее (в разделе 5 ), применив 
описанную вьше технику, мы приведем явный пример однопараметрического семейства орбит для такого действия, переведенный на язык фуксовых особых точек.

Последовательность $1,2,5,16,61$ образует начало известной последовательности последовательности Эйлера-Бернулли $K_{n}$, которая является последовательностью производных в 0 функции sec $x+\operatorname{tg} x$. В работе А. А. Кириллова [13] исследована почти та же задача: над полем из двух элементов им исследовались орбиты действия унитреугольных матриц на нижнетреугольных с нулями на диагонали комбинацией сопряжения и взятия поддиагональной части (коприсоединенное действие). Им получен следуюший ответ: начиная с размерности 6 число орбит начинает отличаться от вьшеупомянутой последовательности, и, более того, если рассматривать аналогичную задачу над полем из $q$ элементов и (некоторьй) $q$-аналог последовательности $K_{n}$, то они также начинают различаться при $n=6$. Это отличие, как нам кажется, может быть вызвано именно наличием однопараметрических семейств дополнительных инвариантов.

\section{5. Примеры дополнительных инвариантов}

\section{1. Пример 1. Рассмотрим две системы:}

$$
\dot{z}=\frac{B_{1}(t)}{t} z, \quad B_{1}(t)=\left(\begin{array}{ccc}
2 & t & 0 \\
0 & 1 & 0 \\
0 & 0 & 0
\end{array}\right)
$$

и

$$
\dot{w}=\frac{B_{2}(t)}{t} w, \quad B_{2}(t)=\left(\begin{array}{ccc}
2 & 0 & 0 \\
0 & 1 & t \\
0 & 0 & 0
\end{array}\right) .
$$

Заметим, что у них одинаковые нормирования (0, 1 и 2), а операторы монодромии сопряжены, однако эти две системы не являются голоморфно эквивалентными: их характеристические матрицы - $\left(\begin{array}{lll}0 & 1 & 0 \\ 0 & 0 & 0 \\ 0 & 0 & 0\end{array}\right)$ и $\left(\begin{array}{lll}0 & 0 & 0 \\ 0 & 0 & 1 \\ 0 & 0 & 0\end{array}\right)$ - не сопряжены никакой верхнетреугольной матрицей.

5.2. Пример 2 и доказательство следствия 2. Предыдущий пример был основан на том, что не только сам оператор монодромии, рассматриваемый с точностью до сопряжения, является инвариантом системы, но также и его ограничение на подпространство решений, имеющих нормирование не меньше $n_{i}$ (для каждого $i$ получаем свой инвариант). Однако это все равно дает дискретную (для каждого набора собственных значений) систему инвариантов. Следующий пример показьвает, что в достаточно большой размерности число классов аналитической сопряженности систем с данным набором собственных значений может быть бесконечньм, что, в частности, означает неполноту вьшеупомянутой системы инвариантов.

ДокАЗАТЕЛЬСТво СЛЕДСТВИЯ 2. Разберем здесь только случай набора собственных значений $0,1, \ldots, 5$ - общий случай ему полностью аналогичен. 
Рассмотрим семейство систем

$$
\dot{z}=\frac{B(t)}{t} z, \quad B(t)=\left(\begin{array}{cccccc}
5 & b_{1} t & b_{2} t^{2} & 0 & 0 & 0 \\
0 & 4 & 0 & 0 & b_{4} t^{3} & 0 \\
0 & 0 & 3 & b_{3} t & 0 & 0 \\
0 & 0 & 0 & 2 & 0 & b_{5} t^{2} \\
0 & 0 & 0 & 0 & 1 & b_{6} t \\
0 & 0 & 0 & 0 & 0 & 0
\end{array}\right),
$$

где все $b_{i} \neq 0$. Их характеристические матрицы имеют вид

$$
A=\widehat{B}(1)=\left(\begin{array}{cccccc}
0 & b_{1} & b_{2} & 0 & 0 & 0 \\
0 & 0 & 0 & 0 & b_{4} & 0 \\
0 & 0 & 0 & b_{3} & 0 & 0 \\
0 & 0 & 0 & 0 & 0 & b_{5} \\
0 & 0 & 0 & 0 & 0 & b_{6} \\
0 & 0 & 0 & 0 & 0 & 0
\end{array}\right) .
$$

Покажем, что никакие две различные матрицы вида (8) не сопряжены унитреугольной матрицей, и, более того, каждая из них является нормальной формой для такого действия. Для этого мы применим технику раздела 4 , упорядочив элементы нильтреугольной матрицы $A$ следуюшим образом:

$$
a_{16}, a_{26}, a_{15}, a_{25}, a_{36}, a_{14}, a_{46}, a_{13}, a_{35}, a_{24}, a_{56}, a_{34}, a_{12}, a_{45}, a_{23} .
$$

Пусть

$$
r_{i_{0} j_{0}}(A)=\operatorname{rank}\left(\left(a_{i j}\right)_{i_{0} \leqslant i, j \leqslant j_{0}}\right) .
$$

Тогда, как легко видеть, $R_{i j, k}(A)=r_{i j}\left(A^{k}\right)$ - инвариант сопряжения унитреугольной (и даже верхнетреугольной) матрицей. Заметим, что поскольку для нашей матрицы $R_{12,1}(A)=R_{34,1}(A)=R_{56,1}(A)=1$, то сопряжением унитреугольной матрицей невозможно обнулить $a_{12}, a_{34}$ и $a_{56}$. Кроме того, поскольку $R_{14,2}(A)=R_{36,2}(A)=1$, $R_{26,1}(A)=2$, не изменив (равные 0 ) $a_{35}$ и $a_{24}$, нельзя обнулить $a_{13}, a_{46}$ или $a_{25}$. Итак, ни один ненулевой элемент нашей матрицы нельзя обнулить, не изменив какой-то из "более старших". Тем самьм наша матрища приведена к нормальной для данного действия форме.

Но лемма 2 утверждает, что в таком случае две матрицы из семейства (8) сопряжены верхнетреугольной матрищей тогда и только тогда, когда они сопряжены диагональной матрицей. С другой стороны, легко видеть, что сопряжение диагональной матрищей не может изменить отношения

$$
\frac{b_{1} b_{4} b_{6}}{b_{2} b_{3} b_{5}}=\frac{a_{12} a_{25} a_{56}}{a_{13} a_{34} a_{46}}
$$

Элементы семейства (8), для которых это отношение различно, не сопряжены никакой верхнетреугольной матрицей. Тем самьм, имеем однопараметрическое семейство попарно не эквивалентных фуксовых особых точек: все системы

$$
\dot{z}=\frac{B_{c}(t)}{t} z, \quad B_{c}(t)=\left(\begin{array}{cccccc}
5 & t & t^{2} & 0 & 0 & 0 \\
0 & 4 & 0 & 0 & c t^{3} & 0 \\
0 & 0 & 3 & t & 0 & 0 \\
0 & 0 & 0 & 2 & 0 & t^{2} \\
0 & 0 & 0 & 0 & 1 & t \\
0 & 0 & 0 & 0 & 0 & 0
\end{array}\right),
$$


c $c \neq 0$ попарно не сопряжены.

Благодарности. Авторы благодарны Ю. С. Ильяшенко за постановку задачи, многократные плодотворные обсуждения и за помощь в написании и редактировании этой статьи, без которой она не могла бы появиться, А. А. Болибруху, благодаря которому в статье появился второй взгляд на рассматриваемую задачу, и Д. В. Алексеевскому, заметившему алгебраический характер задачи. Кроме того, хотелось бы поблагодарить А. С. Городецкого и Э. Жиса за полезные дискуссии и И. В. Аржанщева за обсуждение алгебраической части работы.

\section{СПИСОК ЦИТИРОВАННОЙ ЛИТЕРАТУРЫ}

[1] Арнольд В.И., Ильлшенко Ю.С. Обькновенные дифференциальные уравнения // Итоги науки и техники. Современные проблемы математики. Динамические системы. Т. 1. М.: ВИНИТИ, 1985. С. 7-150.

[2] Levelt A.H. M. Hypergeometric functions, I, II, III, IV // Nederl. Acad. Wetensch. Proc. Ser. A. 1961. V. 64. № 4. P. 361-403.

[3] Гантмахер Ф.Р. Теория матриц. М.: Наука, 1988.

[4] Ильяшенко Ю. С. Нелинейная проблема Римана-Гильберта // Тр. МИАН. 1996. Т. 213. C. 6-29.

[5] Болибрух А. А. Фуксовы дифференциальные уравнения и голоморфные расслоения. М.: МЦНМО, 2000.

[6] Anosov D. V., Bolibrukh A. A. The Riemann-Hilbert Problem. Aspects of Math. Braunschweig: Vieweg, 1994.

[7] Кашин В.В. Орбиты присоединенного и коприсоединенного действия борелевских подгрупп полупростой алгебраической группы // Вопросы теории групп и гомологической алгебры. Ярославль, 1990. С. 141-159.

[8] Коддингтон Э. А., Левинсон Н. Теория обькновенных дифференциальных уравнений. М.: ИЛ, 1958.

[9] Рабинович Б. А. Аналитическая классификация фуксовых особых точек. Дипломная работа. М.: МГУ, 1997.

[10] Болибрух А. А. 21-я проблема Гильберта для линейных фуксовых систем. Тр. МИАН. T. 206. М.: Наука, 1994.

[11] Бродский И. Б. Об инвариантах унипотентных групп // УМН. 1976. Т. 31. № 1. С. 243-244.

[12] Бродский И. Б. Об орбитах унипотентных групп // Функцион. анализ и его прилож. 1969. T. 3. №2. С. 19-23.

[13] Кириллов А. А. Метод орбит и конечные группы // Студенческие чтения МК НМУ. Вып. 1. М.: МЦНМО, 2000. С. 37-73.

(В.А. Клепцьн) Московский государственный

Поступило университет им. М.В. Ломоносова 03.02 .2004 (Б. А. Рабинович) Department of Mathematics, Weizmann Institute of Science, Rehovot, Israel

E-mail: kleptsyn@mccme.ru, borisra@012.net.il 